\title{
Considérations liées à la santé dans le processus de réinstallation des réfugiés syriens au Canada
}

\author{
Hansen $L^{1 *}$, Huston $P^{2}$
}

\section{Résumé}

Le Canada a réagi à l'urgence humanitaire en Syrie en s'engageant à accueillir 25000 réfugiés syriens avant le début de l'année 2016. Il s'agissait d'une entreprise complexe, qui a nécessité une coordination avec différents organismes internationaux, comme le Haut Commissariat des Nations Unies pour les réfugiés (HCR), l'Organisation internationale pour les migrations (OIM) et les ministères fédéraux, y compris Immigration, Réfugiés et Citoyenneté Canada (IRCC), l'Agence des services frontaliers du Canada (ASFC), le ministère de la Défense nationale (MDN) et l'Agence de la santé publique du Canada (ASPC). Au Canada, cette initiative a demandé la collaboration des administrations provinciales et municipales, d'organisations non gouvernementales et de bénévoles, dont des répondants du secteur privé, pour planifier la transition des réfugiés syriens vers leur nouvelle vie au Canada.

Dans leurs préparatifs pour recevoir les réfugiés syriens, les organismes gouvernementaux ne prévoyaient pas d'être confrontés à de graves menaces de maladies infectieuses. Toutefois, les bilans récents de l'expérience de l'Europe en matière d'accueil des réfugiés et de celle des fournisseurs de soins de santé qui offrent des services à d'autres populations de réfugiés donnent à penser que cette population a des besoins de santé non comblés et des problèmes de santé non traités, découlant des migrations qui ont cours depuis trois à quatre ans. Dans cet esprit, de grands efforts de planification ont été déployés pour faire face aux défis potentiels en matière de santé publique. Fournisseurs de services sociaux et interprètes médicaux se sont proposés pour aider les Syriens à accéder au système de soins de santé et expliquer leurs besoins. Les communautés de pratique au Canada ont répondu en fournissant des soins, ainsi qu'en élaborant et en mettant à jour des outils et des ressources, en faveur d'une approche adaptée à la culture et fondée sur des données probantes, dans le but d'évaluer les besoins de santé des réfugiés syriens, et y répondre.

\section{Affiliations}

'Direction générale de l'infrastructure de sécurité sanitaire, Agence de la santé publique du Canada, Ottawa (ON)

${ }^{2}$ Direction générale de la prévention et du contrôle des maladies infectieuses, Agence de la santé publique du Canada, Ottawa (ON)

*Correspondance : Lisa.Hansen@ phac-aspc.gc.ca

Citation proposée : Hansen L, Huston P. Considérations liées à la santé dans le processus de réinstallation des réfugiés syriens au Canada. Relevé des maladies transmissibles au Canada 2016;42-Suppl 2:S3-8.

https://doi.org/10.14745/ccdr.v42is2a02f

\section{Introduction}

La crise de réfugiés syriens est une urgence humanitaire complexe, que l'on définit comme une perturbation sociopolitique grave, qui a une incidence sur la capacité d'une population à combler ses besoins fondamentaux (nourriture, eau, abris et sécurité physique) (1). Depuis 2011, la Syrie est impliquée dans une guerre civile, compliquée par la montée de différents groupes rebelles et du groupe État islamique en Irak et en Syrie (EIIS). Selon les estimations, près de 250000 civils ont été tués, et environ la moitié de la population du pays, qui compte 22 millions de personnes, a été déplacée (2). En novembre 2015, le Haut Commissariat des Nations Unies pour les réfugiés (HCR) avait relevé presque quatre millions de réfugiés syriens (3) : près de deux millions en Turquie, et plus de deux millions en Égypte, en Irak, en Jordanie et au Liban. Les autres avaient été déplacés en Syrie ou avaient fui en Afrique du Nord et en Europe.
Devant cette urgence humanitaire, le gouvernement du Canada s'est engagé, à l'automne 2015, à réinstaller 25000 réfugiés syriens avant le début de l'année 2016, dans le cadre de l'«Opération réfugiés syriens ». Le présent article, qui met l'accent sur les besoins en matière de santé connus et prévus, décrit le plan du gouvernement pour identifier, évaluer et réinstaller les réfugiés syriens qui immigrent au Canada. II aborde également certains défis connus, liés à la prestation de services de santé aux réfugiés, et les stratégies à envisager pour y faire face. Un article d'accompagnement décrit l'accueil des réfugiés syriens, et fournit l'information sur leur état de santé initial (4).

\section{Opération réfugiés syriens}

La réinstallation sûre et efficace de milliers de réfugiés est une entreprise complexe, qui suppose une coordination avec des gouvernements étrangers, des organismes internationaux, comme le Haut Commissariat des Nations Unies pour les réfugiés 
(HCR), I'Organisation internationale pour les migrations (OIM) et de multiples ministères du gouvernement canadien, parmi lesquels Immigration, Réfugiés et Citoyenneté Canada (IRCC), l'Agence des services frontaliers du Canada (ASFC), le ministère de la Défense nationale (MDN) et l'Agence de la santé publique du Canada (ASPC), ainsi que les administrations provinciales et municipales, les organisations non gouvernementales et les bénévoles, dont plusieurs répondants du secteur privé, issus de groupes confessionnels et d'organismes communautaires.

\section{Stratégie de réinstallation}

La mise en œuvre de la stratégie de réinstallation comporte cinq phases:

\section{Identification les réfugiés syriens qui arriveront au Canada}

Le Haut Commissariat des Nations Unies pour les réfugiés était chargé de l'enregistrement, des vérifications de sécurité et de la sélection des réfugiés syriens provenant de sites de réfugiés situés au Liban et en Jordanie. Le gouvernement de la Turquie a identifié les réfugiés vivant à l'intérieur de ses frontières. Les entrevues de sécurité et d'immigration ont été effectuées par des agents des visas chevronnés. Le Canada a convenu d'accorder la priorité aux réfugiés les plus vulnérables, représentant un risque moindre pour la sécurité : femmes à risque, familles complètes et personnes considérées comme étant vulnérables en raison de leur auto-identification en tant que personnes lesbienne, gaie, bisexuelle, transgenre et intersexuée (LGBTI) (5).

\section{Traitement des demandes des Syriens à l'étranger}

Une fois le filtrage de sécurité terminé, les réfugiés à destination du Canada ont subi un examen médical réglementaire, effectué par des médecins certifiés; il s'agit d'une exigence standard imposée à tous les immigrants. La pratique courante veut qu'un candidat à l'immigration soit interdit de territoire pour motifs sanitaires si son état de santé constitue vraisemblablement un danger pour la santé ou la sécurité publiques ou risque d'entraîner un fardeau excessif pour les services sociaux ou de santé (6).

Dans le cadre de l'Opération réfugiés syriens, l'examen médical réglementaire servait surtout d'outil de dépistage visant à déterminer les besoins de soins de santé et à établir leur ordre de priorité. Les résultats de cet examen médical ont été traités de manière confidentielle et communiqués aux réfugiés, sous la forme, notamment, d'une copie papier des résultats de l'examen, accompagnés des consultations de suivi recommandées, au besoin. Si un candidat était admissible, mais que l'examen médical réglementaire avait détecté une maladie infectieuse à déclaration obligatoire, comme le $\mathrm{VIH}$, la syphilis ou une tuberculose latente, IRCC informait l'autorité de la santé de la localité ou de la région du Canada où le demandeur était censé s'installer.

\section{Transport vers le Canada}

Après les vérifications de sécurité et de l'état de santé, et la délivrance d'un visa de sortie, les réfugiés syriens devaient se rendre au Canada en empruntant, principalement, deux types de vols. Il s'agissait, dans la majeure partie des cas, de vols nolisés spéciaux, à destination de Toronto et de Montréal, et, dans une plus faible proportion de cas, de vols commerciaux vers d'autres points d'entrée internationaux au Canada. Avant de s'envoler, chaque voyageur a subi une évaluation de son aptitude à prendre l'avion, effectuée par l'Organisation internationale pour les migrations et d'autres partenaires. Cette évaluation, réalisée tout juste avant le départ, visait à déterminer la présence d'un problème de santé émergent, susceptible d'empêcher le voyage (par exemple, dans le cas d'une grossesse très avancée ou d'une femme en travail) et à faciliter la préparation du niveau de soins de santé approprié à fournir, au besoin, à l'arrivée (7). Une escorte de l'Organisation internationale pour les migrations était à bord de tous les vols nolisés transportant des réfugiés à destination du Canada.

La plupart des réfugiés n'avaient pas de problèmes médicaux évidents; certains avaient besoin de soins de santé courants et quelques autres, de soins médicaux rapides à leur arrivée.

\section{Accueil au Canada}

Tous les réfugiés arrivés à bord d'un avion nolisé à l'aéroport international Pearson de Toronto et à l'aéroport Pierre-ElliottTrudeau de Montréal ont été accueillis en tant que résidents permanents; ils ont fait l'objet d'un contrôle afin que soit détecté tout signe de maladie et ont reçu la documentation du Programme fédéral de santé intérimaire. Les agents de I'ASPC ont effectué les vérifications de sécurité frontalière habituelles.

Les voyageurs qui semblaient malades à leur arrivée ont été évalués par un agent de quarantaine de I'ASPC. Après avoir terminé l'évaluation, l'agent de quarantaine déterminait les mesures à prendre. En fonction de l'évaluation et des critères de la Loi sur la mise en quarantaine, un voyageur pouvait être appelé à se rapporter à l'autorité de santé publique locale, subir une évaluation médicale plus poussée ou se rendre là où il était attendu. Lorsque cela était indiqué, les réfugiés étaient dirigés immédiatement vers des équipes médicales d'urgence, fournies par les autorités provinciales de la santé de l'Ontario et du Québec, et dépêchées dans les centres d'accueil près des aéroports. Les équipes médicales d'urgence étaient sur place pour évaluer et traiter les problèmes de santé et, dans la mesure du possible, pour éviter des transferts inutiles à l'hôpital. En outre, elles étaient là pour faire la coordination avec les soins cliniques et les services de santé publique locaux, le cas échéant (8). 5. Installation et intégration à la communauté

\section{Installation et intégration à la communauté}

Une fois dépassé le point d'entrée, les réfugiés syriens ont commencé à rejoindre leur collectivité d'accueil. Dans la plupart des cas, après les longues heures passées en avion, l'établissement au Canada a commencé par un bref séjour dans un hôtel situé à proximité de l'aéroport. Les réfugiés parrainés par le secteur privé ont été dirigés vers leurs répondants et les réfugiés parrainés par le gouvernement ont été dirigés vers l'un des 30 à 35 programmes communautaires d'aide à la réinstallation partout au pays. Une fois arrivés dans leur collectivité d'accueil, certains réfugiés sont restés temporairement dans des hôtels locaux, jusqu'à ce qu'ils trouvent un hébergement permanent. Des sites de logement temporaire, sur les bases des Forces canadiennes au Québec et en Ontario, financés par les ministères provinciaux de la Santé, la Société canadienne de la Croix-Rouge et l'Agence de la santé publique du Canada $(9,10)$, ont également été désignés à titre 
de plan de contingence pour l'hébergement des réfugiés. Des services de santé de transition, comme les cliniques de soins dentaires et les programmes de vaccination de rattrapage, ont été organisés par les autorités sanitaires locales et les systèmes de soins de santé des provinces. Les organismes communautaires de réinstallation des réfugiés ont appuyé l'intégration des réfugiés en offrant de la formation linguistique et des services sociaux, ainsi qu'en inscrivant les enfants aux écoles locales. Le Centre des opérations du portefeuille de la Santé, géré par l'Agence de la santé publique du Canada, a coordonné les volets de l'intervention fédérale en ce qui concerne la santé et a fait la liaison avec les ministères de la Santé des provinces et des territoires. Ces derniers appuient la coordination des soins dans leur territoire respectif.

La figure 1 schématise la succession d'évaluations auxquelles se soumettent les réfugiés, et les services qui leur sont offerts, à partir du processus d'identification et d'évaluation au Moyen-Orient jusqu'à l'intégration dans les collectivités canadiennes.

\section{Soins et services de santé offerts aux réfugiés en voie de se réinstaller}

Les soins et les services de santé offerts aux réfugiés en voie de se réinstaller comprennent, notamment, une évaluation médicale fondée sur des données probantes et l'intégration des réfugiés au système de santé (11). En tant que résidents permanents, la plupart des nouveaux arrivants syriens étaient admissibles immédiatement à l'assurance-maladie provinciale, étant donné que la plupart des provinces ont levé l'exigence de la période d'attente de trois mois. En outre, les nouveaux arrivants bénéficient, jusqu'à un an, de la couverture du Programme fédéral de santé intérimaire du gouvernement du Canada. On a demandé aux professionnels de la santé de s'inscrire auprès de Croix Bleue Medavie pour fournir des produits et des services de santé aux bénéficiaires du Programme fédéral de santé intérimaire. Ce programme offre aux réfugiés et à leur famille une couverture supplémentaire, qui inclut certains services et produits, comme les soins dentaires, les soins de la vue et les médicaments sur ordonnance. (12).

Les soins et les services de santé pour les réfugiés en voie de se réinstaller ont été organisés à l'échelle provinciale et à l'échelle locale dans l'ensemble du pays. En Ontario, par exemple, où environ 10000 réfugiés ont été réinstallés, le gouvernement provincial a activé le Centre des opérations d'urgence du ministère pour apporter sa collaboration et partager de I'information avec les différents ordres de gouvernement et les partenaires locaux; il a en outre créé le Plan d'action ontarien pour le système de santé et les réfugiés syriens (13), lequel comprenait la Ligne Info-santé pour les réfugiés, qui met les réfugiés en contact avec des fournisseurs de soins de santé, ainsi qu'une adresse électronique générique où envoyer les demandes de renseignements. Les réseaux locaux d'intégration des services de santé (RLISS) ont aidé à la planification et à la coordination locale des services de santé. Par exemple, le réseau local de Champlain a contribué à mettre sur pied un groupe de travail sur la santé des réfugiés, composé de représentants de la Ville d'Ottawa (y compris Santé publique Ottawa), de centres de santé communautaires, d'hôpitaux et d'organismes d'aide à la réinstallation de la région. Le groupe de travail a élaboré un plan, dont faisait partie l'Ottawa Newcomer Clinic, qui a servi de centre de dépistage, chargé de faire l'évaluation initiale des réfugiés et d'offrir de la vaccination et des soins dentaires. Une fois que les réfugiés étaient installés dans un logement permanent, ils étaient mis en contact avec un médecin de famille dans la communauté pour les soins primaires courants.

\section{Soins primaires}

Au Canada, les réseaux de professionnels de la santé fournissant des soins aux réfugiés, comme la Collaboration canadienne pour la santé des immigrants et des réfugiés, ont été renforcés au cours des dernières années (14). Des ressources pour les cliniciens ont été créées, y compris un guide de pratique axé sur les patients pour les soins de santé primaires prodigués aux réfugiés syriens (11), une liste de vérification clinique fondée sur des données probantes, créée par la Collaboration canadienne pour la santé des immigrants et des réfugiés (15),

Figure 1 : Évaluations auxquelles se soumettent les réfugiés syriens en provenance du Moyen-Orient jusqu'à leur arrivée au Canada

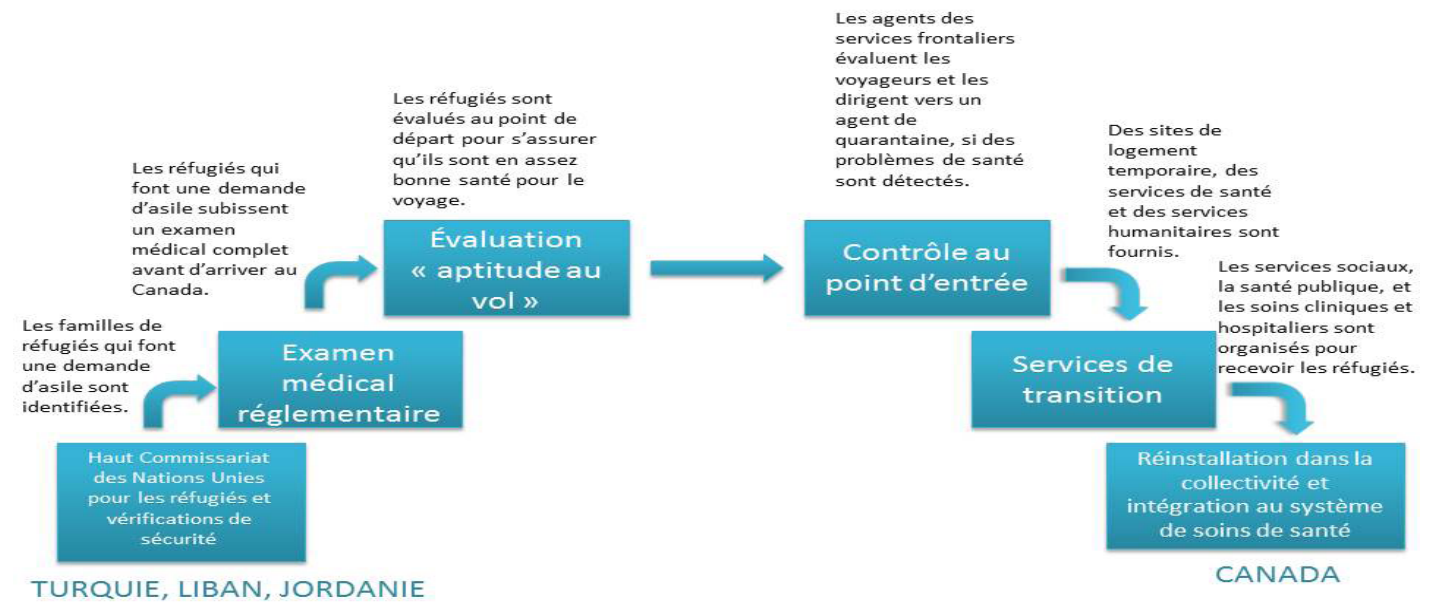


des ressources s'adressant précisément aux enfants, créées par la Société canadienne de pédiatrie (16), et des ressources en santé mentale, commandées par le Haut Commissariat des Nations Unies pour les réfugiés, s'adressant aux Syriens (17).

\section{Santé publique}

Les autorités de santé publique locales et régionales ont travaillé avec les fournisseurs de soins de santé pour répondre au besoin en matière de vaccination de rattrapage des réfugiés syriens. La surveillance de routine est en place et peut être améliorée, au besoin, pour évaluer les répercussions de l'arrivée d'un grand nombre de nouveaux arrivants dans le système de santé, détecter toute maladie infectieuse émergente, et intervenir (13). Les ministères provinciaux ont tenu les professionnels de la santé informés des questions liées à la santé à mesure qu'elles survenaient, comme le besoin de soins dentaires urgents.

\section{Défis communs}

Selon le rapport du Haut Commissariat des Nations Unies pour les réfugiés, la majorité des réfugiés syriens ont fait preuve d'une remarquable résilience et se rétablissent rapidement, une fois que leurs besoins de base en matière de logement, de nourriture et de sécurité étaient comblés (16). Néanmoins, on s'attend à ce qu'un petit pourcentage de réfugiés au Canada ait des besoins complexes ou multiples en matière de santé. Selon l'expérience antérieure de grandes migrations de réfugiés au Canada et ailleurs, la prestation efficace des soins de santé aux réfugiés sera inévitablement complexe.

Les nouveaux arrivants syriens auront comme difficulté importante de veiller à régler leurs problèmes de santé et de mettre leur santé au premier plan, à un moment où ils sont préoccupés par leur réinstallation, laquelle inclut l'intégration à la collectivité et la recherche d'un logement, l'intégration à l'école et dans les cercles sociaux. Pour veiller à ce que le système de santé soit prêt à répondre à leurs besoins en matière de santé, des réseaux de fournisseurs de soins de santé et de spécialistes de la santé des réfugiés ont commencé à entrer en contact avec les cliniciens, pour leur donner des conseils sur les problèmes de santé attendus, y compris les problèmes de santé mentale. Il est également nécessaire d'offrir un soutien approprié à ces services, comme les services d'interprètes médicaux capables de traduire la terminologie médicale, mais aussi d'être témoins de la douleur et de la souffrance que vivent les réfugiés. À certains moments, les interprètes ne sont disponibles que par téléphone. Le recours à des interprètes ayant une formation interculturelle est associé à une confiance plus grande, de la part des fournisseurs de soins de santé, quant aux soins qu'ils fournissent aux réfugiés, un meilleur dépistage des problèmes lors de l'évaluation et une augmentation de la satisfaction de la clientèle (18).

Selon le rapport du Haut Commissariat des Nations Unies pour les réfugiés, de nombreux réfugiés syriens ont vécu de la détresse psychologique et sociale après avoir vécu dans une zone de guerre, et du fait d'avoir été déplacés de leur domicile et d'avoir à s'installer dans un nouveau pays (17). Bon nombre d'entre eux ont perdu des membres de leur famille et des amis, et certains ont été témoins ou victimes de violence. Cela peut entraîner des réactions émotionnelles (p. ex., tristesse, deuil, colère), des symptômes physiques (p. ex., fatigue, insomnie) et des troubles somatiques, ou des problèmes sociaux et comportementaux (p. ex. retrait ou agressivité) (17). Ces expériences peuvent inclure la difficulté à trouver un logement approprié et du travail, et à surmonter les obstacles linguistiques, la discrimination et l'isolement social (19-21).

Les Syriens, tout comme les autres nouveaux immigrants, peuvent être parfois réticents à discuter de leur sentiment d'isolement ou de leur détresse, en particulier dans un nouvel environnement. On recommande actuellement aux fournisseurs de soins de santé de s'attaquer activement aux problèmes de santé, et non d'investiguer les traumatismes - car cela pourrait empirer les choses (11) - et d'être attentifs aux signes de trouble de stress post-traumatique, de dépression et d'autres problèmes de santé mentale, qui peuvent se manifester des mois après I'arrivée au Canada.

Pour répondre aux problèmes de santé mentale des réfugiés syriens, différents niveaux, ou dimensions, de soutien et de services sont recommandés (17). Le niveau de base consiste à se pencher sur les déterminants sociaux de la santé mentale, comme un logement sécuritaire et adéquat. Le deuxième niveau consiste à évaluer et à optimiser les différentes formes de soutien de la part de la famille et de la collectivité, qui favorisent la cohésion au sein de la population de réfugiés. Dans certains cas, du soutien psychosocial ciblé est nécessaire pour aider les réfugiés à faire face à leur situation et à s'adapter au Canada. Ces trois niveaux contribueront à régler la plupart des problèmes de santé mentale. Si un problème de santé mentale grave survient, il est recommandé d'offrir des services de santé mentale adaptés sur le plan culturel; la ressource du Haut Commissariat des Nations Unies pour les réfugiés donne des renseignements utiles sur la façon de fournir ces services (17).

Les fournisseurs de soins de santé peuvent se sentir dépassés devant les difficultés que représentent les besoins en matière de soins de santé des réfugiés. Bon nombre de fournisseurs de soins de santé sont déjà débordés et les problèmes de santé des réfugiés constituent un problème supplémentaire, qui exige de la préparation et du temps pour offrir les soins. En dépit des bonnes intentions et du nombre croissant de ressources disponibles, la demande sera élevée, et il se peut que les fournisseurs de soins de santé et d'autres personnes éprouvent une usure de compassion. II est possible de la voir venir et d'y faire face en optimisant le recours aux ressources de soutien offertes par les organismes et les organisations professionnelles, les outils en ligne et la télésanté, les groupes de soutien formels et informels et les contacts avec des organismes ou $d$ 'autres personnes, qui ont une expertise en résilience et connaissent bien les besoins de santé mentale des personnes qui interviennent en situation de crise.

\section{Conclusion}

Les réfugiés syriens qui viennent au Canada ont pris la décision difficile de quitter leur pays millénaire, autrefois dynamique, que la guerre a détruit. Le Canada accueille les réfugiés par l'entremise d'un système de collaboration complexe, qui fait appel à des organismes internationaux, à plusieurs ministères fédéraux et provinciaux et administrations municipales, à des organisations non gouvernementales et à des milliers de bénévoles. Du point de vue de la santé, la priorité consiste à déterminer les besoins de santé physique et mentale des 
nouveaux arrivants et de créer des services accessibles et adaptés sur le plan culturel pour répondre à ces besoins.

Les nouveaux arrivants syriens font face à des difficultés pour surmonter le traumatisme que représente le fait d'avoir vécu une urgence humanitaire et l'adaptation à la vie au Canada. Les fournisseurs de soins de santé et d'autres fournisseurs de services font face eux aussi à des difficultés lorsque vient le temps de répondre aux besoins des réfugiés syriens. Grâce à la planification, la coordination, les ressources, le soutien mutuel et les systèmes en place pour déterminer les nouveaux problèmes à mesure qu'ils surviennent, tous les éléments sont là pour accueillir les réfugiés syriens et les aider efficacement à se réinstaller au Canada.

\section{Remerciements}

Nous souhaitons remercier toutes les personnes qui travaillent à l'échelle internationale, nationale, régionale et locale dans le monde entier pour aider à redonner aux réfugiés syriens un lieu de vie sûr.

Nota : La Dre Huston est la rédactrice scientifique en chef du Relevé des maladies transmissibles au Canada et se récuse des décisions de la rédaction relatives au présent article. Un grand merci à la $D^{\text {re }}$ Lee Lior, pour avoir assumé le rôle de rédactrice en chef aux fins du présent article.

\section{Conflit d'intérêts}

Aucun.

\section{Références}

1. Gushalak BD, MacPherson DW, Prochazka H, Cooper MM. The practice of immigration halth in complex emergency situations - A case study from Kosovo from March to July 1999. Refuge. 2000;18:46-51.

2. Citizenship and Immigration Canada. Population profile: Syrian refugees. Ottawa, ON: CIC; November 2015. (Disponible en français : http://www.cpa.ca/docs/File/ Cultural/FR\%20Syrian\%20Population\%20Profile.pdf).

3. United Nations High Commission for Refugees. Syria Regional Refugee Response. Inter-agency information sharing portal Geneva, SW; United Nations; 2015. http:// data.unhcr.org/syrianrefugees/regional.php.

4. Hansen L, Maidment L, Ahmad R. Early observations on the health of Syrian refugees in Canada. Can Comm Dis Rep 2016;42-Suppl 2:S8-10. (Disponible en français : www.phacaspc.gc.ca/publicat/ccdr-rmtc/16vol42/dr-rm42-S2/ar-03-fra. php).
5. Government of Canada. \#WelcomeRefugees: Security and health screening Ottawa, ON: Government of Canada; 2016. (Disponible en français : http://www.cic.gc.ca/francais/ refugies/bienvenue/survol/securite.asp).

6. Citizenship and Immigration Canada. The immigration medical assessment. Ottawa, ON: Government of Canada; 2016. (Disponible en français : http://www.cic.gc.ca/francais/ ressources/outils/medic/eval/utiliser.asp).

7. International Organization for Migration. Migration health annual review 2014 Geneva, SW: IOM; 2015. http:// publications.iom.int/books/migration-health-annualreview-2014.

8. Bhatia N, Sarwal S, Robinson H, Geduld J, Huneault F, et.al. Federal public health strategies to minimize the importation of communicable diseases into Canada. Can Comm Dis Rep. 2015 Suppl 6;41:S3-8. (Disponible en français : http://www. phac-aspc.gc.ca/publicat/ccdr-rmtc/15vol41/dr-rm41s-6/ assets/pdf/15vol41s-6-fra.pdf).

9. National Defence and the Canadian Armed Forces. Operation PROVISION Ottawa, ON: Government of Canada; 2016. (Disponible en français : http://www.forces.gc.ca/fr/ operations-etranger/op-provision.page).

10. Canadian Red Cross. Syrian refugee crisis and refugee arrival in Canada. Ottawa, ON: Canadian Red Cross; 2016. (Disponible en français : http://www.croixrouge.ca/noschamps-d-action/services-aux-migrants-et-aux-refugies/ crise-des-refugies-en-syrie-et-accueil-des-refugies-syriensau-canada?lang=fr-CA\&_ga=1.98459487.1512838470.1457 969009).

11. Pottie K, Greenaway C, Hassan G, Hui C, Kirmayer LJ. Caring for a newly arrived Syrian refugee family. Can Med Assoc J. 2015; preprint Dec 8, 2015.

12. Citizenship and Immigration Canada. Interim Federal Health Program. IFHP information handbook for health care professionals. Ottawa, ON: CIC; 2006. www.medavie. bluecross.ca/cs/BlobServer?blobcol=urldata\&blobtable= MungoBlobs\&blobheadervalue2=abinary; +charset=UTF8\&blobheadername2=MDT-Type\&blobkey=id\&blobwhe re $=1187213290372 \&$ blobheader=application/pdf.

13. Ontario Ministry of Health and Long-Term Care. Ontario Health System Action Plan: Syrian refugees. Toronto, ON: MOHLTC; December 17, 2015. (Disponible en français : http://www.health.gov.on.ca/fr/pro/programs/emb/ syrianrefugees/docs/health_system_action_plan.PDF).

14. Canadian Collaboration for Immigrant and Refugee Health (CCIRH) Migrant Health Knowledge Exchange Network. Working together to improve the health of migrants. Ottawa, ON: CCIRH; 2015. http://www.ccirhken.ca/index. html.

15. Canadian Collaboration for Immigrant and Refugee Health $(\mathrm{CCIRH})$. Evidence-based clinical e-checklist for immigrants. Ottawa, ON: CCIRH; 2015. http://www.ccirhken.ca/ccirh/ checklist_website/index.html.

16. Canadian Pediatric Society. Caring for kids new to Canada website. Ottawa, ON: CPS; 2015. http://www. kidsnewtocanada.ca/. 
17. Hassan G, Kirmayer LJ, Mekki-Berrada A, Quosh C, el Chammay R, Deville-Stoetzel, et al. Culture, context and the mental health and psychosocial wellbeing of Syrians: A review for mental health and psychosocial support staff working with Syrians affected by armed conflict. Geneva, SW: UNHCR, 2015. http://mhpss.net/?get=250/Culture_ mental-health_Syrians-FINAL1.pdf.

18. Joshi C, Russell G, Cheng IH, Kay M, Pottie K, Alston M, et al. A narrative synthesis of the impact of primary health care delivery models for refugees in resettlement countries on access, quality and coordination. Int J Equity Health. 2013 Nov 7;12:88. http://www.ncbi.nlm.nih.gov/pmc/articles/ PMC3835619/pdf/1475-9276-12-88.pdf.

19. Campbell M. Social determinants of mental health in new refugees in the UK: Cross-sectional and longitudinal analyses. Lancet. 2012;380:S27.
20. Wilson RM, Murtaza R, Shakya YB Pre-migration and post-migration determinants for newly arrived refugees in Toronto. Canadian Issues: Immigrant Mental Health. Summer 2010:45-50.

21. Mawani F. Social determinants of refugee mental health. In Simich L, Andermann L (eds.) Refuge and resilience: Promoting resilience and mental health among resettled refugees and forced migrants (International Perspectives on Migration, Vol. 7). Berlin: Springer Science + Business Media; 2014. 